\title{
DIETA LOW CARB COMO ESTRATÉGIA DE MANEJO NA REMISSÃO DO DIABETES MELLITUS INSULINORRESISTENTE: SÍNTESE DE EVIDÊNCIAS
}

\author{
Low carb diet as a management strategy in the remission of Diabetes Mellitus type II: synthesis of \\ evidences
}

\author{
Wagner Sessa ${ }^{1}$, Renato Ribeiro Nogueira Ferraz ${ }^{2}$
}

${ }^{1}$ Nutricionista. Universidade Nove de Julho (UNINOVE) - São Paulo, SP. ORCID: https://orcid.org/0000-0002-6745-0669. E-mail: wsessa@gmail.com. ${ }^{2}$ Biólogo. Doutor em Ciências Básicas com Pós-Doutorado em Ciência da Informação. Professor do Mestrado Profissional em Administração - Gestão em Sistemas de Saúde (PMPA-GSS). Universidade Nove de Julho (UNINOVE) - São Paulo, SP. ORCID: https://orcid.org/0000-0002-8931-895X. E-mail: renatobio@hotmail.com

\begin{abstract}
Resumo
Introdução: O DM tipo II está associado à deficiência no funcionamento de determinados mecanismos responsáveis pela regulação da sensibilidade tecidual à ação da insulina, em concomitância à secreção deficiente do hormônio pelas células beta pancreáticas. As dietas LC restringem os carboidratos diários para algo entre 20 e $50 \mathrm{~g}$, mostrando associação com uma considerável perda de peso. Todavia, os efeitos da dieta LC no controle do DM II são bastante controversos. Objetivo: Realizar uma revisão da literatura englobando ensaios clínicos que comprovadamente testaram os efeitos da dieta LC na remissão do DM II. Método: Para seleção dos trabalhos foi utilizada a seguinte estratégia de busca: (low-carb OR "low carb" OR "low carbohydrate" OR low-carbohydrate OR ketogenic OR Atkins) AND (diabetes OR diabetics) AND (type-2 OR "type 2" OR type-II OR "type II" OR "insulinresistant") AND "clinical trial", na base de dados PUBMED/MEDLINE. Resultados: Foram revisados 12 artigos que preencheram os critérios de inclusão. Síntese de Evidências: A dieta LC melhora o equilíbrio glicêmico, devendo ser considerada em pacientes portadores de DM tipo II e que necessitam de suporte nutricional. A referida dieta ainda reduz a gordura visceral, melhora a sensibilidade à insulina e aumenta os níveis de HDL. A estabilidade da glicemia diurna e do perfil lipídico podem ser mantidos com a dieta LC, sem efeitos adversos renais, e sem a necessidade de medicamentos hipoglicemiantes. A dieta é bem tolerada e se mostra bastante segura, mesmo quando prescrita em longo prazo.
\end{abstract}

Palavras-chave: Gestão em Saúde; Low carb; Nutrição; Diabetes; Tratamento.

\begin{abstract}
Introduction: Type II DM is associated with a deficiency in the mechanisms responsible to the regulation of tissue sensitivity to insulin action, in concomitance with deficient secretion of the hormone by pancreatic beta cells. LC diets restrict daily carbohydrates to anything between 20 and $50 \mathrm{~g}$, showing association with considerable weight loss. However, the effects of the LC diet on the control of DM II are quite controversial. Objective: To perform a literature review encompassing clinical trials that have proven the effects of the LC diet on the remission of DM II. Method: The following search strategy was used: (low-carb OR "low carb" OR "low carbohydrate" OR lowcarbohydrate OR ketogenic OR Atkins) AND (type-2 OR "type 2" OR type-II OR "type II" OR
\end{abstract}


insulin-resistant) AND "clinical trial" in the PUBMED / MEDLINE database. Results: Twelve articles that met the inclusion criteria were reviewed. Summary of Evidences: The LC diet improves glycemic balance and should be considered in patients with type II DM who require nutritional support. Said diet further reduces visceral fat, improves insulin sensitivity and increases HDL levels. The stability of diurnal glycaemia and lipid profile can be maintained with the LC diet, without renal adverse effects, and without the need for hypoglycemic drugs. The diet is well tolerated and is quite safe, even when prescribed in the long period.

Keywords: Health Management; Low carb; Nutrition; Diabetes; Treatment.

\section{Introdução}

Ainda em meados da década de 1930, já era discutida a hipótese de que o complexo de sintomas associados ao Diabetes Mellitus (DM) poderia ser decorrente de duas entidades patogênicas distintas. Uma delas estaria associada à deficiência na produção de insulina, um hormônio hipoglicemiante, caracterizando o DM sensível à insulina (tipo I). Já a segunda forma, caracterizada pela ausência de um fator até então desconhecido, e que por sua vez tornava os tecidos sensíveis à insulina, se referia ao DM insulinorresistente (tipo II) (HIMSWORTH, 1936).

Normalmente, logo após a refeição há um aumento nos níveis sanguíneos de glicose, o que por sua vez estimula a secreção de insulina, resultando em um aumento no transporte, biotransformação e armazenamento da glicose nos músculos e tecido adiposo. Deficiência na produção de insulina, ou mesmo resistência aos seus efeitos, resulta em absorção tecidual reduzida de glicose, com consequente hipoglicemia intracelular e hiperglicemia extracelular. A hipoglicemia intracelular causa a gliconeogênese, que induz a quebra das gorduras corporais gerando uma condição de cetoacidose diabética. Esta condição leva à redução da síntese proteica e da produção de gamaglobulinas, causando caquexia, polifagia e comprometimento da cicatrização, ao mesmo tempo em que a hiperglicemia extracelular causa prejuízos aos vasos e à circulação sanguínea, eleva sobremaneira a diurese osmótica e pode evoluir para o estado de coma hiperglicêmico (ASMAT; ABAD; ISMAIL, 2016; KANGRALKAR; PATIL; BANDIVADEKAR, 2010; OZOUGWU et al., 2013).

No DM tipo I, a deficiência na secreção de insulina devido à destruição autoimune das células beta pancreáticas, leva a distúrbios metabólicos diversos cujo estágio final representa o início clínico da doença. A autoimunidade, além da composição genética e de fatores ambientais estão diretamente associados ao desenvolvimento da doença. Já o DM tipo II está associado à defíciência no funcionamento de determinados mecanismos responsáveis pela regulação da sensibilidade tecidual à ação da insulina, em concomitância à secreção deficiente do hormônio pelas células beta pancreáticas. Neste tipo de diabetes, múltiplos defeitos genéticos e certos fatores ambientais, especialmente a obesidade, são os principais responsáveis pelo início das alterações etiopatogênicas responsáveis pela instalação da doença (ASMAT; ABAD; ISMAIL, 2016).

Apesar dos bilhões de dólares gastos a cada ano em dietas e produtos para perda de peso e melhoria das condições de saúde, com destaque especial para o controle do DM II, poucas pessoas conseguem o sucesso ao iniciar programas populares de dieta. Uma dieta que tem levantado preocupações de segurança entre a comunidade científica desde o início da década de 2000 é a dieta com reduzido teor de carboidratos (low carb) (TAPPER-GARDZINA; COTUGNA; VICKERY, 2002). As dietas LC restringem os carboidratos diários para algo entre 20 e $50 \mathrm{~g}$, de forma semelhante 
ao que acontece com as dietas clínicas cetogênicas, mostrando associação com uma considerável perda de peso (GIUGLIANO et al., 2018).

Os efeitos da dieta LC sobre o estado geral de pacientes com DM tipo I é bastante discutível. Uma recente revisão da literatura que buscou compilar todas as evidências até então disponíveis relacionadas ao tema, concluiu que este possível efeito ainda não está completamente esclarecido, e que estudos primários que avaliem os efeitos de curto e longo prazos das dietas LC ainda são necessários para apoiar seu uso na prática (TURTON; RAAB; ROONEY, 2018). De forma semelhante, os efeitos da dieta LC no controle do DM II também são bastante controversos. Um também recente artigo de opinião, publicado em importante periódico internacional relatou que, para indivíduos com diagnóstico de DM II, os riscos de um descontrole dos níveis glicêmicos, normalmente associado ao consumo excessivo de carboidratos, supera em muito os riscos à saúde atribuídos às gorduras saturadas, e a maioria das pessoas com DM II deve se concentrar na limitação de carboidratos, particularmente carboidratos de cadeia simples, como prioridade.

Face à controvérsia sobre o tema, e também com base na inexistência de literatura nacional que aborde o tema em profundidade, a presente revisão de literatura pretende avaliar os ensaios clínicos que comprovadamente testaram os efeitos da dieta LC no controle do DM II. Acredita-se que, com a realização desta pesquisa, será fornecido material compilado e recente, e que por sua vez poderá contribuir para que o nutricionista tome a melhor decisão clínica em relação à prescrição de dietas LC para pacientes portadores de DM II.

\section{Objetivo}

Realizar uma revisão da literatura englobando apenas os ensaios clínicos que comprovadamente testaram os efeitos da dieta LC na remissão do DM II.

\section{Método}

Este artigo descreve uma pesquisa de caráter exploratório, baseado no método de revisão da literatura com síntese das principais evidências clínicas relacionadas ao tema proposto. Para seleção dos trabalhos foi utilizado o motor de busca da base de dados PUBMED/MEDLINE, ao pertencente à National Library of Medicine, do National Institutes of Health dos Estados Unidos.

A busca dos trabalhos foi realizada com base na seguinte estratégia: (low-carb OR "low carb" OR "low carbohydrate" OR low-carbohydrate OR ketogenic OR Atkins) AND (diabetes OR diabetics) AND (type-2 OR "type 2" OR type-II OR "type II" OR "insulin-resistant") AND "clinical trial". Somente artigos científicos foram considerados para a revisão. Foram excluídos da lista de trabalhos as teses de Doutorado, dissertações de Mestrado, Trabalhos de Conclusão de Cursos de Especialização e Graduação, resumos e trabalhos completos publicados em anais de eventos, materiais informativos, livros e capítulos de livros. Eventualmente, artigos que não discutiam diretamente os efeitos da dieta low carb na remissão do Diabetes Mellitus tipo II foram também excluídos do estudo.

Os trabalhos selecionados foram inseridos em uma ferramenta automatizada de gestão de referencial, denominada Zotero (FERRAZ, 2016; ZOTERO, 2019), que é um software gratuito. Em seguida, foram apresentados na seção de Revisão da Literatura por ordem cronológica de publicação. 


\section{Resultados}

A busca pelas referências foi realizada no dia 04 de junho de 2019. Inicialmente foram identificados 55 itens, conforme demonstrado na Figura 1.

Format: Summary - Sort by: Best Match - Per page: 100 -
Search results
Items: 55

Figura 1: Resultados da busca inicial na base de dados PUBMED/MEDLINE. Fonte: https://www.ncbi.nlm.nih.gov/pubmed

Em seguida, os trabalhos foram inseridos em uma biblioteca digital, quando então seus títulos foram lidos. Nesse momento, foram excluídos 43 artigos que não discutiam especificamente os efeitos da dieta LC na remissão do DM tipo II, como por exemplo, trabalhos que testavam os efeitos de medicamentos, além daqueles que avaliavam a dieta LC em situações muito específicas, como no pós-operatório de cirurgias bariátrica, dentre outros. Alguns artigos também foram excluídos por se tratarem de estudos experimentais. Os 12 artigos que resistiram a essa primeira avaliação são apresentados na Figura 2.

\begin{tabular}{|c|c|c|c|}
\hline Título & Autor & Ano & A Publicação \\
\hline > The effect of different nutritional feeds on the postprandial glucose response in healthy volunteers and patients with type II diabetes & Hofman et al. & 2004 & European Journal ... \\
\hline > Beneficial effect of low carbohydrate in low calorie diets on visceral fat reduction in type 2 diabetic patients with obesity & Miyashita et al. & 2004 & Diabetes Research... \\
\hline > Low-carbohydrate and high-fat intake among adult patients with poorly controlled type 2 diabetes mellitus & Ma et al. & 2006 & Nutrition (Burbank... \\
\hline > Beneficial effects of ketogenic diet in obese diabetic subjects & Dashti et al. & 2007 & Molecular and Cell... \\
\hline > Comparative study of the effects of a 1-year dietary intervention of a low-carbohydrate diet versus a low-fat diet on weight and glycemic control in type $2 \ldots$. & Davis et al. & 2009 & Diabetes Care \\
\hline > Effect of low-calorie versus low-carbohydrate ketogenic diet in type 2 diabetes & Hussain et al. & 2012 & Nutrition (Burbank... \\
\hline > A randomized cross-over trial of the postprandial effects of three different diets in patients with type 2 diabetes & Fernemark et al. & 2013 & Plos One \\
\hline > Improvements in glucose metabolism and insulin sensitivity with a low-carbohydrate diet in obese patients with type 2 diabetes & Krebs et al. & 2013 & Journal of the Am... \\
\hline > A randomized pilot trial of a moderate carbohydrate diet compared to a very low carbohydrate diet in overweight or obese individuals with type 2 diabet... & Saslow et al. & 2014 & Plos One \\
\hline Short-term safety, tolerability and efficacy of a very low-calorie-ketogenic diet interventional weight loss program versus hypocaloric diet in patients with t... & & 2016 & Nutrition \& Diabet.. \\
\hline > F Third Exposure to a Reduced Carbohydrate Meal Lowers Evening Postprandial Insulin and GIP Responses and HOMA-IR Estimate of Insulin Resistance & Lin e Borer & 2016 & Plos One \\
\hline n energy-restricted low-carbohydrate, high unsaturated fat/low saturated fat diet versus a high-carbohydrate, low- & & 2018 & Diabetes, Obesity ... \\
\hline
\end{tabular}

Figura 2: Artigos que resistiram à primeira avaliação.

Fonte: dados obtidos pelos autores com o software Zotero.

A segunda etapa de seleção consistiu na leitura dos resumos dos 12 artigos previamente selecionados. Após a leitura, verificou-se que um estudo não se tratava de um ensaio clínico, mas apenas de um trabalho observacional (MA et al., 2006), justificando a sua exclusão. Dessa forma, os 11 artigos restantes foram lidos na íntegra, resumidos, e apresentados na próxima seção em ordem cronológica de publicação.

\section{Revisão da Literatura}

Hofman e colaboradores (2004), realizaram um estudo cruzado, duplo-cego e randomizado, cujo objetivo foi avaliar a resposta glicêmica pós-prandial a quatro dietas diferentes com relação à composição em macronutrientes e fibras. No estudo foram incluídos 20 pacientes, sendo 10 voluntários saudáveis e 10 pacientes com DM tipo II. Os participantes receberam $200 \mathrm{kcal}$ no formato de quatro produtos dietéticos, sendo dois produtos padrão e dois produtos específicos para o DM tipo 
II. A glicose plasmática em jejum e no período pós-prandial foi avaliada por duas horas, sendo calculados o pico de glicemia e a área sob a curva glicêmica. Como principais resultados os autores identificaram que a dieta LC, com alto teor de ácidos graxos monoinsaturados e alto conteúdo de fibras, diminuiu significativamente o pico de concentração de glicose e área sob a curva glicêmica, em comparação aos alimentos com composição padrão. A conclusão dos autores foi que a dieta LC rica em ácidos graxos monoinsaturados e fibras melhora o equilíbrio glicêmico e, portanto, deve ser considerada em pacientes diabéticos que necessitam de suporte nutricional.

Miyashita et al (2004), objetivaram investigar os efeitos da dieta LC no metabolismo de glicose e lipídios, especialmente no acúmulo de gordura visceral, em comparação a uma dieta rica em carboidratos. Para tal, indivíduos obesos com DM tipo II foram aleatoriamente designados para

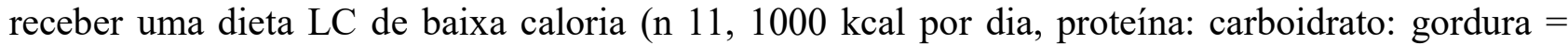
25:40:35), ou uma dieta LC $(n=11,1000 \mathrm{kcal}$ por dia, proteína: carboidrato: gordura $=25: 65: 10)$, por 4 semanas. Dentre os resultados observados os autores ressaltaram que ocorrerão diminuições similares no peso corporal e nos níveis séricos de glicose em ambos os grupos. Já os níveis séricos de insulina em jejum foram reduzidos no grupo LC em comparação com o grupo com dieta rica em carboidratos $(-30 \%$ versus $-10 \%, \mathrm{P}<0,05)$. O colesterol sérico total e os níveis de triglicérides diminuíram em ambos os grupos, mas não foram significativamente diferentes um do outro. $\mathrm{O}$ colesterol de alta densidade (HDL-C) aumentou no grupo com dieta LC, mas não no grupo com dieta rica em carboidratos $(+15 \%$ versus $0 \%, \mathrm{P}<0,01)$. Houve uma diminuição maior na área de gordura visceral, medida pela tomografia computadorizada, no grupo LC em comparação com o grupo de dieta rica em carboidratos (-40 cm (2) versus $-10 \mathrm{~cm}(2), \mathrm{P}<0,05)$. Todavia, a proporção de área de gordura visceral para área de gordura subcutânea não se alterou no grupo com dieta rica em carboidratos (de 0,70 para 0,68 ), mas diminuiu significativamente no grupo LC (de 0,69 para $0,47, \mathrm{P}$ $<0,005)$. Em conclusão, os autores afirmaram que a dieta restrita LC se mostrou como um tratamento efetivo para redução da gordura visceral, melhora da sensibilidade à insulina e aumento dos níveis de HDL-C em indivíduos portadores de DM tipo II.

Em seu estudo, Dashti e colaboradores (2007) avaliaram o efeito da dieta cetogênica em indivíduos obesos com hiperglicemia, comparando-os a indivíduos normoglicêmicos. Para tal, 64 obesos saudáveis com índice de massa corporal (IMC) maior que 30 e hiperglicêmicos, foram comparados aos seus controles normoglicêmicos. Em ambos os grupos, foram avaliados antes do início do protocolo, e após 8, 16, 24, 48 e 56 semanas, o peso corporal, IMC, glicemia, colesterol total, LDL-colesterol, HDL-colesterol, triglicerídeos, ureia e creatinina. Os resultados demonstraram que o peso corporal, IMC, nível de glicose sanguínea, colesterol total, LDL-colesterol, triglicerídeos e ureia reduziram significativamente, comparando a semana 1 com a semana $56(\mathrm{P}<0,0001)$, enquanto o nível de HDL-colesterol aumentou significativamente $(p<0,0001)$. Curiosamente, estas alterações foram mais significativas em indivíduos com níveis elevados de glicose no sangue, em comparação com aqueles com níveis normais de glicose. Os autores finalizaram o artigo afirmando que o estudo demonstrou os efeitos benéficos em longo prazo da dieta cetogênica em obesos diabéticos, sendo a dieta LC é segura mesmo quando prescrita em longo prazo.

O grupo de Davis (2009), buscou comparar os efeitos de uma dieta LC e reduzida em gorduras na perda de peso e controle glicêmico de pacientes com DM tipo II, pelo período de um ano. Para tal, conduziram um ensaio clínico randomizado com 105 adultos com sobrepeso e DM tipo II. Nesses pacientes foram avaliados a hemoglobina glicada, a pressão arterial e o perfil lipídico aos 3, 6 e 12 meses de protocolo. Dentre os achados, observou-se maior redução no peso e da hemoglobina glicada 
apenas nos primeiros 3 meses. A perda de peso ocorreu mais rapidamente no grupo LC quando comparado ao grupo sob dieta apenas com baixo teor de gordura $(p=0,005)$, embora após 12 meses a uma redução de peso tenha sido similar. Embora não tenham sido observadas mudanças nos valores da pressão arterial, houve um aumento maior no HDL no grupo LC $(p=0,002)$. Os autores finalizaram o estudo ressaltando que, entre os pacientes com DM tipo II, após 1 ano, uma dieta LC trouxe como efeito benéfico apenas aumento no HDL.

Hussain et al (2012), conduziram um ensaio cujo objetivo foi compreender os efeitos benéficos da LC em comparação a uma dieta hipocalórica (LCD) no controle da glicemia. Sendo assim, 363 pacientes com sobrepeso e obesidade foram recrutados para um estudo com duração de 24 semanas. Do total, 102 pacientes eram diabéticos tipo II. Os participantes foram aconselhados a escolher o LCD ou o LC, dependendo de sua preferência. O peso corporal, índice de massa corporal, alterações na circunferência da cintura, glicemia, hemoglobina e hemoglobina glicada, colesterol total, lipoproteína de baixa densidade, lipoproteína de alta densidade, triglicerídeos, ácido úrico, ureia e creatinina foram determinados antes do início do protocolo, e após 4, 8, 12, 16, 20 e 24 semanas de administração das dietas. A avaliação dos resultados permitiu aos autores destacar que tanto a LCD quanto a LC apresentaram efeitos benéficos em todos os parâmetros analisados. Curiosamente, essas mudanças foram mais significativas em indivíduos que estavam sob dieta LC, em comparação com aqueles no LCD. Dessa forma, os autores concluíram que existem efeitos benéficos em ambas as dietas oferecidas, embora estes tenham sido mais pronunciados nos indivíduos portadores de DM tipo II que receberam a dieta LC.

Fernemark et al (2013), compararam por meio de um estudo cruzado e randomizado, uma dieta com baixo teor de gordura (45-56\% de energia vinda dos carboidratos), e uma dieta LC (16$24 \%$ de energia vinda dos carboidratos), com uma dieta de estilo mediterrâneo. Dos 21 pacientes portadores de DM tipo II inicialmente recrutados, 19 completaram o estudo. Segundo os pesquisadores, foi possível detectar que a dieta LC reduziu a liberação de insulina e o nível de glicose sérica em comparação com a dieta com baixo teor de gordura $(\mathrm{p}<0,0005)$. Ainda, a resposta à insulina após a refeição do almoço ao estilo mediterrânico foi mais pronunciada do que durante o almoço com baixo teor de gordura (aumento da insulina na dieta com baixo teor de gordura: 4,35 $\pm 2,2$, da dieta mediterrânica: $8,12 \pm 5,2 \mathrm{p}=0,001$ ), embora os níveis de glicose pós-prandial tenham sido semelhantes. Ao final do protocolo, os autores concluíram que a refeição no estilo mediterrâneo induziu elevações na glicose pós-prandial semelhantes à refeição com baixo teor de gordura, apesar da quantidade quase dobrada de calorias, devido a um pronunciado aumento de insulina. Isto sugere que ingestão calórica decorrente do café da manhã e do almoço, em comparação a uma refeição única no estilo mediterrâneo, pode ser vantajosa em uma perspectiva metabólica.

Krebs e colaboradores (2013), examinaram os efeitos de uma dieta LC e rica em gorduras, com base na avaliação da sensibilidade à insulina, controle glicêmico e fatores de risco para doença cardiovascular. Para tal, 14 pacientes obesos (IMC: 40,6 $\pm 4,9 \mathrm{~kg} / \mathrm{m}^{2}$ ), portadores de DM tipo 2, foram recrutados para receber a dieta LC estilo "Atkins". As avaliações foram realizadas com $0,12 \mathrm{e}$ 24 semanas de protocolo, e se referiram à determinação do peso, da sensibilidade à insulina, da hemoglobina glicada, dos lipídios e da pressão arterial. Os 12 pacientes que finalizaram o estudo perderam uma média de $9,7 \pm 1,8 \mathrm{~kg}$ de peso ao longo das 24 semanas, perda essa atribuível a uma grande redução nos carboidratos e à consequente redução no consumo total de energia. $\mathrm{O}$ controle glicêmico melhorou significativamente, e a glicemia de jejum, a avaliação do modelo de homeostase e a área sob a curva de glicose foram significativamente reduzidos na semana $12(\mathrm{p}<0,05)$. Ainda, 
houve redução da pressão arterial sistólica, e a lipoproteína de alta e baixa, além do colesterol total, se mostraram aumentados, embora a relação HDL e triglicerídeos tenha reduzido. A conclusão final do trabalho foi que a dieta LC foi bem tolerada e reduziu significativamente o peso corporal em pacientes diabéticos, além de permitir o controle glicêmico sem a necessidade de agentes hipoglicemiantes.

Saslow et al (2014), compararam em seu estudo os efeitos de duas dietas sobre a hemoglobina glicada $(\mathrm{HbA} 1 \mathrm{c})$, além de outros parâmetros relacionados à saúde em adultos com sobrepeso ou obesos, portadores de DM tipo II ou pré-diabéticos. Em seu estudo randomizado, os autores dividiram os pacientes de forma que um dos grupos recebesse uma dieta com baixo teor de gordura, médio teor de carboidratos e restrição de calorias (MCCR), consistente com as diretrizes da American Diabetes Association ( $\mathrm{n}=18)$, e o outro grupo recebesse uma dieta bastante reduzida em carboidratos, alta em gorduras, e sem restrição calórica ( $\mathrm{LCK}, \mathrm{n}=16$ ). Ambos os grupos se reuniram por 13 sessões, durante 3 meses, e receberam informações sobre dieta e sobre suas habilidades psicológicas para promover mudanças em seu comportamento alimentar. Dentre os resultados observou-se que, aos 3 meses, o nível médio de HbA1c se manteve inalterado desde o início do estudo no grupo MCCR, enquanto diminuiu $0,6 \%$ no grupo LCK; houve diferença significativa entre os grupos na alteração da HbA1c, favorecendo o grupo LCK $(-0,6 \%$, IC $95 \%,-1,1 \%$ a $-0,03 \%, p=0,04)$. Do total, $44 \%$ dos participantes do grupo LCK interromperam um ou mais medicamentos para diabetes, em comparação com apenas $11 \%$ do grupo MCCR ( $\mathrm{p}=0,03)$; o grupo LCK perdeu em média 5,5 kg vs. 2,6 kg perdidos no grupo MCCR $(\mathrm{p}=0,09)$. Em suma, a conclusão dos autores foi que uma dieta muito baixa em carboidratos, juntamente com o estímulo das habilidades para promoção de mudanças de comportamento, pode melhorar o controle glicêmico do DM tipo II, permitindo inclusive a diminuição do uso de medicamentos para tratamento do DM.

Goday et al (2016), em um programa de perda de peso intervencionista, e por meio de um ensaio clínico prospectivo, aberto, multicêntrico, randomizado e com duração de 4 meses, avaliaram a segurança e a tolerabilidade em curto prazo de uma dieta LC $(<50 \mathrm{~g}$ de carboidratos por dia), incluindo o estilo de vida e o suporte para modificação comportamental, conhecido como Método Diaprokal. O protocolo foi constituído por 89 homens e mulheres, com idades entre 30 e 65 anos, portadores de DM tipo II, e com IMC entre 30 e $35 \mathrm{~kg} \mathrm{~m}^{2}$. Os grupos foram aleatoriamente, sendo um deles para perda de peso intervencionista (dieta LC) e o outro para receber a dieta padrão de baixa caloria. Com relação aos resultados, não houve diferenças significativas nos parâmetros de segurança laboratorial quando comparados os dois grupos. Alterações na relação albumina / creatinina na dieta LC não foram significativas quando comparadas ao grupo controle. A creatinina e a ureia no sangue não se alteraram significativamente em relação à linha de base, tão pouco entre os grupos. Perda de peso e redução da circunferência da cintura no grupo dieta LC foram significativamente maiores do que nos controles (ambos $\mathrm{p}<0,001$ ). O declínio na HbA1c e o controle glicêmico foi maior no grupo LC ( $\mathrm{p}<0,05)$. Com base nesses resultados, os autores afirmaram que o programa de perda de peso intervencionista baseado na dieta LC é mais eficaz na redução do peso corporal e na melhora do controle glicêmico do que uma dieta hipocalórica padrão, com segurança e boa tolerância para pacientes com DM tipo II.

Lin e Borer (2016), avaliaram quatro grupos de 8 mulheres na pós-menopausa com peso equivalente e metabolicamente saudáveis, que receberam três refeições isocalóricas (uma refeição antes da avaliação, e duas refeições durante o dia de teste), contendo $30 \%$ ou $60 \%$ de carboidratos, com e sem duas horas de exercício moderado antes das duas últimas refeições. Como resultados, 
observou-se que a terceira refeição com baixo teor de carboidratos, mas não a refeição com alto teor de carboidrato, reduziu: (1) a área da curva glicêmica noturna em 39\% sem exercício, e em 31\% após o exercício; (2) a secreção de peptídeo insulinotrópico em $48 \%$ sem exercício e $45 \%$ após o exercício; e (3) a resistência à insulina à noite em $37 \%$ sem exercício e em $24 \%$ após o exercício. Dessa forma, foi possível aos pesquisadores concluírem que a insulina pós-prandial noturna, as respostas do peptídeo insulinotrópico e a resistência à insulina diminuíram em mais de $30 \%$ após as refeições que limitaram a ingestão diária de carboidratos para 30\%, em comparação com essas mudanças após as refeições com $60 \%$ de carboidrato, um efeito independente do exercício pré-refeição. Ainda, o exercício pré-refeição exacerbou a intolerância à glicose com ambas as dietas, provavelmente devido ao comprometimento da sinalização da insulina.

Por fim, Tay et al (2018) conduziram um estudo para examinar se uma dieta LC, com alto teor de gordura insaturada / baixa saturação seria capaz de melhorar o controle glicêmico e os fatores de risco para doença cardiovascular (DCV) em pacientes com sobrepeso e obesos com DM tipo II. O estudo avaliou 115 adultos com que foram randomizados para receber dietas hipocalóricas combinadas com exercícios aeróbicos / resistidos ( 1 hora, 3 dias / semana) por 2 anos: O grupo LC recebeu dieta contendo $14 \%$ de energia como carboidrato, $28 \%$ como proteína e $58 \%$ como gordura ( $<10 \%$ de gordura saturada); o outro grupo recebeu dieta com baixo teor de gordura, alto teor de carboidrato e baixo índice glicêmico, sendo 53\% como carboidrato, $17 \%$ como proteína e $30 \%$ como gordura $(<10 \%$ de gordura saturada). Em todos os pacientes foram avaliados, antes e após a intervenção, a HbAlc, variabilidade glicêmica $(\mathrm{GV})$, escore de efeito da medicação anti-glicêmica (MES, calculado com base na potência e dosagem do medicamento para DM), peso, composição corporal, DCV e marcadores de risco renal. Após a avaliação dos inúmeros resultados, os autores concluíram que ambas as dietas alcançaram perda de peso comparável, além de reduções de HbA1c. No entanto, a dieta LC sustentou maiores reduções nas necessidades de medicação para o DM tipo II, gerando ainda mais estabilidade da glicemia diurna e do perfil lipídico, sem efeitos adversos renais, sendo considerada eficaz no tratamento do DM tipo II.

\section{Síntese de Evidências}

Em suma, com base na síntese das informações apresentadas nos estudos avaliados, é fato que a dieta LC melhora o equilíbrio glicêmico, devendo ser considerada em pacientes portadores de DM tipo II e que necessitam de suporte nutricional. A referida dieta ainda reduz a gordura visceral, melhora a sensibilidade à insulina e aumenta os níveis de HDL. A estabilidade da glicemia diurna e do perfil lipídico podem ser mantidos com a dieta LC, sem efeitos adversos renais, e sem a necessidade de medicamentos hipoglicemiantes. A dieta é bem tolerada e se mostra bastante segura, mesmo quando prescrita em longo prazo. Todavia, deve-se estimular os pacientes a promoverem mudanças em seu próprio comportamento alimentar.

\section{Referências}

ASMAT, U.; ABAD, K.; ISMAIL, K. Diabetes mellitus and oxidative stress-A concise review. Saudi Pharmaceutical Journal, v. 24, n. 5, p. 547-553, 2016.

DASHTI, H. M. et al. Beneficial effects of ketogenic diet in obese diabetic subjects. Molecular and Cellular Biochemistry, v. 302, n. 1-2, p. 249-256, 2007. 
DAVIS, N. J. et al. Comparative study of the effects of a 1-year dietary intervention of a lowcarbohydrate diet versus a low-fat diet on weight and glycemic control in type 2 diabetes. Diabetes Care, v. 32, n. 7, p. 1147-1152, 2009.

FERNEMARK, H. et al. A randomized cross-over trial of the postprandial effects of three different diets in patients with type 2 diabetes. PloS One, v. 8, n. 11, p. e79324, 2013.

FERRAZ, R. R. N. Como inserir citações e listar as referências do meu trabalho acadêmico de maneira automatizada? Redação Científica, Princípios de Estatística e Bases de Epidemiologia para simples mortais. Erechim: Deviant, 2016. p. 313.

GIUGLIANO, D. et al. More sugar? No, thank you! The elusive nature of low carbohydrate diets. Endocrine, v. 61, n. 3, p. 383-387, 2018.

GODAY, A. et al. Short-term safety, tolerability and efficacy of a very low-calorie-ketogenic diet interventional weight loss program versus hypocaloric diet in patients with type 2 diabetes mellitus. Nutrition \& Diabetes, v. 6, n. 9, p. e230, 2016.

HIMSWORTH, H. P. Diabetes mellitus. Its differentiation into insolin-sensitive and insulininsensitive types. Lancet, v. 230, p. 127-130, 1936.

HOFMAN, Z. et al. The effect of different nutritional feeds on the postprandial glucose response in healthy volunteers and patients with type II diabetes. European Journal of Clinical Nutrition, v. 58, n. 11, p. 1553-1556, 2004.

HUSSAIN, T. A. et al. Effect of low-calorie versus low-carbohydrate ketogenic diet in type 2 diabetes. Nutrition (Burbank, Los Angeles County, Calif.), v. 28, n. 10, p. 1016-1021, 2012.

KANGRALKAR, V. A.; PATIL, S. D.; BANDIVADEKAR, R. M. Oxidative stress and diabetes: a review. International Journal of Pharmaceutical Applications, v. 1, n. 1, p. 38-45, 2010.

KREBS, J. D. et al. Improvements in glucose metabolism and insulin sensitivity with a lowcarbohydrate diet in obese patients with type 2 diabetes. Journal of the American College of Nutrition, v. 32, n. 1, p. 11-17, 2013.

LIN, P.-J.; BORER, K. T. Third Exposure to a Reduced Carbohydrate Meal Lowers Evening Postprandial Insulin and GIP Responses and HOMA-IR Estimate of Insulin Resistance. PloS One, v. 11, n. 10, p. e0165378, 2016.

MA, Y. et al. Low-carbohydrate and high-fat intake among adult patients with poorly controlled type 2 diabetes mellitus. Nutrition (Burbank, Los Angeles County, Calif.), v. 22, n. 11-12, p. 1129-1136, 2006.

MIYASHITA, Y. et al. Beneficial effect of low carbohydrate in low calorie diets on visceral fat reduction in type 2 diabetic patients with obesity. Diabetes Research and Clinical Practice, v. 65, n. 3, p. 235-241, 2004.

OZOUGWU, J. C. et al. The pathogenesis and pathophysiology of type 1 and type 2 diabetes mellitus. Journal of Physiology and Pathophysiology, v. 4, n. 4, p. 46-57, 2013.

SASLOW, L. R. et al. A randomized pilot trial of a moderate carbohydrate diet compared to a very low carbohydrate diet in overweight or obese individuals with type 2 diabetes mellitus or prediabetes. PloS One, v. 9, n. 4, p. e91027, 2014.

TAPPER-GARDZINA, Y.; COTUGNA, N.; VICKERY, C. E. Should you recommend a low-carb, high-protein diet? The Nurse Practitioner, v. 27, n. 4, p. 52, 2002.

TAY, J. et al. Effects of an energy-restricted low-carbohydrate, high unsaturated fat/low saturated fat diet versus a high-carbohydrate, low-fat diet in type 2 diabetes: A 2-year randomized clinical trial. Diabetes, Obesity \& Metabolism, v. 20, n. 4, p. 858-871, 2018. 
TURTON, J. L.; RAAB, R.; ROONEY, K. B. Low-carbohydrate diets for type 1 diabetes mellitus: A systematic review. PLOS ONE, v. 13, n. 3, p. e0194987, 2018.

ZOTERO. Your personal research assistant. Disponível em: <https://www.zotero.org/>. Acesso em: 3 dez. 2018. 